\title{
Kalman Filter-Based Channel Tracking in MIMO-OSTBC Systems
}

\author{
Murilo B. Loiola, Renato R. Lopes, and João M. T. Romano \\ DSPCom lab, School of Electrical and Computer Engineering, University of Campinas. \\ Avenida Albert Einstein, 400 - CP 6101, CEP 13083-852, Campinas, Brazil. \\ E-mails: \{mloiola, rlopes\}@decom.fee.unicamp.br; romano@dmo.fee.unicamp.br.
}

\begin{abstract}
In this paper we propose low-complexity algorithms for estimating flat, time-varying and spatially correlated MIMO channels. The proposed estimators employ Kalman filters to track the channel in orthogonal space-time block coded systems. After developing a state-space model for spatially correlated MIMO channels, we show that the proposed estimators can be simplified by using the orthogonality inherent to orthogonal space-time block codes. We also show that the channel estimates provided by the proposed algorithms correspond to weighted sums of instantaneous maximum likelihood channel estimates. For constant modulus signal constellations, we reduce the receiver complexity even more by proposing a steady-state Kalman filter. Simulation results indicate that there is no significant performance degradation of the steady-state filter compared to the first proposed algorithm and that both algorithms outperform conventional adaptive filters.
\end{abstract}

\section{INTRODUCTION}

Space-time codes are an effective and practical way to exploit spatial diversity in multiple-input, multipleoutput (MIMO) systems, allowing the system to benefit from transmit diversity without any power increment and with no channel knowledge at the transmitter. Among the existing space-time coding schemes, orthogonal space-time block codes (OSTBCs) [1]-[4] are of particular interest because they achieve full diversity at low receiver complexity. More specifically, the maximum likelihood (ML) receiver for OSTBCs consists of a linear receiver followed by a symbol-by-symbol decoder.

In order to correctly decode the received signals, the ML decoder for OSTBCs must have perfect channel knowledge. Unfortunately, this channel information is not normally available to the receivers; therefore channel estimation techniques are essential for the system to work properly. When the channel is static, methods such as those presented in [3][5] can be successfully used. However, the channel may be time-varying due to the mobility of the transmitter and/or receiver, to changes in the environment, or to carrier frequency mismatch between transmitter and receiver. In these cases, the estimation algorithm must be able to track the channel variations. One of the most widely known approaches to channel tracking is Kalman filtering [6]-[9]. In the literature

This work was supported by Fundação de Amparo a Pesquisa do Estado de São Paulo (FAPESP) under grant $05 / 55310-8$ and by Conselho Nacional de Desenvolvimento Científico e Tecnológico (CNPq), under grant $311844 / 2006-5$. of MIMO systems, a Kalman filter (KF) is used to estimate channels in space-time trellis coded systems in [8], while channel estimation in MIMO-OFDM systems is performed by a Kalman filter in [9].

The use of Kalman filters to estimate channels in space-time block coded systems is also developed in the literature. In [10], a KF is used to estimate fast flat fading MIMO channels in Alamouti-based schemes. Therefore, it is limited to the case of two transmit antennas. An extension of [10] for any type of OSTBCs is presented in [11]. It is also shown in [11] that the $\mathrm{KF}$ can be significantly simplified due to the orthogonality of OSTBC codewords.

The algorithms developed in [10], [11] assume that the channel coefficients are uncorrelated and independent from each other. Furthermore, the complexity reduction in [11] cannot be achieved if the channels were correlated. However, spatial correlation between transmit and/or receive antennas usually exists in practical scenarios [12]. This can occur, for instance, if the separation of adjacent antennas is not sufficient to allow the signals undergo different channel fades.

The first contribution of this paper is a Kalman filter-based estimator for flat, time-varying and spatially correlated MIMO channels. Thanks to the use of OSTBCs, the classical KF can be simplified, leading to an algorithm of reduced complexity. The algorithms in [10] and [11] can be seen as special cases of the method proposed in this work, when the spatial correlation matrix is diagonal. We also show that the proposed algorithm corresponds to a filter that estimates the channel as a weighted sum of previous estimates and instantaneous maximum likelihood estimates.

Although the proposed KF is less complex than the conventional implementation of the $\mathrm{KF}$, it still needs a matrix inversion at each iteration. However, in the simulations we observe that the inverse converges quickly. Taking advantage of this fact, the second contribution of this paper is the proposition of a steady-state Kalman filter for constant modulus signal constellations. The steady-state Kalman filter computes the asymptotic value of the inverse only once, at the initialization of the algorithm, keeping it constant for all subsequent calculations. The proposed steady-state KF has just a fraction of the complexity of the first proposed algorithm and also generates estimates by weighted averages of instantaneous maximum likelihood channel estimates. We also show by 
simulations that this simple algorithm suffers negligible performance degradation compared to the first proposed algorithm.

The remainder of the paper is organized as follows: section II presents the system model, briefly describing orthogonal space-time block codes and developing an autoregressive model to spatially correlated and time-varying MIMO channels. In section III we detail the proposed reduced complexity Kalman channel estimator, while in section IV we develop the steady-state Kalman filter. Simulation results are shown in section V. Finally, section VI concludes the paper.

\section{SYSTEM MODEL}

We consider a MIMO system with $N_{T}$ transmit antennas sending data blocks of length $T$ to $N_{R}$ receive antennas. The channel is assumed to be flat and constant during the transmission of each data block and can change between consecutive blocks. The relationship between transmitted and received signals for a data block $k$ can be expressed as [3]

$$
\mathbf{Y}_{k}=\mathbf{H}_{k} \mathbf{X}_{k}+\mathbf{N}_{k},
$$

where $\mathbf{Y}_{k}$ is an $N_{R} \times T$ matrix with the received signals, $\mathbf{X}_{k}$ is an $N_{T} \times T$ matrix containing the transmitted signals at block $k$, the $N_{R} \times T$ matrix $\mathbf{N}_{k}$ contains samples of independent, zero mean, circularly symmetric, white Gaussian noise with covariance $\sigma_{n}^{2}$, and the frequency-flat channel is represented by the $N_{R} \times N_{T}$ matrix $\mathbf{H}_{k}$.

In space-time block coding, the matrix $\mathbf{X}_{k}$ represents a mapping that transforms a block of $M$ complex symbols, $\mathbf{x}_{k}=\left[\begin{array}{llll}x_{k, 1} & x_{k, 2} & \cdots & x_{k, M}\end{array}\right]^{\top}$, to an $N_{T} \times T$ complex matrix. The space-time codeword $\mathbf{X}_{k}$ is then used to transmit these $M$ symbols in $T$ time slots, achieving a rate of $M / T$. The matrix $\mathbf{X}_{k}$ is an OSTBC if [2]-[4]: 1) all elements of $\mathbf{X}_{k}$ are linear functions of symbols of $\mathbf{x}_{k}$ and their complex conjugates and 2) for an arbitrary $\mathbf{x}_{k}$, the matrix $\mathbf{X}_{k}$ satisfies $\mathbf{X}_{k} \mathbf{X}_{k}^{\mathrm{H}}=\left\|\mathbf{x}_{k}\right\|^{2} \mathbf{I}_{N_{T}}$, where $\mathbf{I}_{N_{T}}$ is the identity matrix of order $N_{T},\|\cdot\|$ represents the Euclidean norm and $(\cdot)^{\mathrm{H}}$ denotes the conjugate transpose of a matrix.

One of the main characteristics of OSTBCs is the simplicity of the decoder. More specifically, the ML space-time decoder for OSTBCs consists of a linear receiver, which generates sufficient statistics through a linear combination of the received signals, followed by a symbol-by-symbol decision device [2][4]. It is important to highlight that the computation of the sufficient statistics depends on the channel knowledge at the receiver.

As mentioned before, the channel is assumed to be fixed during the transmission of a space-time codeword $\mathbf{X}_{k}$, but can vary between consecutive codewords. According to the widely used wide-sense stationary uncorrelated scattering (WSSUS) model [13], the channel coefficients are modeled as independent, zero-mean, complex Gaussian random variables with time autocorrelation function

$$
\mathrm{E}\left[h_{k, i, j} h_{t, i, j}^{*}\right] \approx \mathcal{J}_{0}\left(2 \pi f_{D} T_{s}|k-t|\right),
$$

where $h_{k, i, j}, i=1, \ldots, N_{R}, j=1, \ldots, N_{T}$ is the $(i, j)$ element of matrix $\mathbf{H}_{k}, \mathcal{J}_{0}$ is the zero-order Bessel function of the first kind, $f_{D} T_{s}$ is the normalized Doppler rate (assumed the same for all transmit-receive antenna pairs) and $T_{s}$ is the baud duration.

Although exact modeling of channel dynamics by finite length autoregressive (AR) processes is impossible because the time autocorrelation function (2) is nonrational and its spectrum is bandlimited, we can approximate the time evolution of channel coefficients by low-order AR processes [8][11]. Therefore, following [8]-[11], we herein approximate the MIMO channel variations by a first order AR process. Thus, defining $\operatorname{vec}(\cdot)$ as the operator that stacks the columns of a matrix on top of each other, the time evolution of the channel is given by

$$
\mathbf{h}_{k}=\beta \mathbf{h}_{k-1}+\mathbf{w}_{k},
$$

where $\mathbf{h}_{k}=\operatorname{vec}\left(\mathbf{H}_{k}\right), \beta=\mathcal{J}_{0}\left(2 \pi f_{D} T_{s}\right), \mathbf{w}_{k}$ is a vector of length $N_{R} N_{T}$ containing independent samples of circularly symmetric, zero-mean, Gaussian excitation noise with covariance matrix $\mathbf{Q}=\sigma_{w}^{2} \mathbf{I}_{N_{R} N_{T}}$, and $\sigma_{w}^{2}=\left(1-\beta^{2}\right) P_{k}$, with $P_{k}=\mathrm{E}\left[\left|h_{k, m}\right|^{2}\right], m=1, \ldots, N_{R} N_{T}$.

In general, however, the channel coefficients present a spatial correlation that depends on the propagation environment, the polarization of the antenna elements and the spacing between them. Among the spatially correlated channel models, one of the most used splits the fading correlation into two independent components called receive correlation and transmit correlation, respectively. This amounts to modeling the channel as [3], [12]

$$
\mathbf{H}_{k}^{\mathrm{c}}=\mathbf{R}_{R}^{1 / 2} \mathbf{H}_{k}\left(\mathbf{R}_{T}^{1 / 2}\right)^{\top},
$$

where $\mathbf{R}_{R}$ models the correlation between receive antennas, $\mathbf{R}_{T}$ models the correlation between transmit antennas, $(\cdot)^{1 / 2}$ stands for the Hermitian square root of a matrix [14] and $\mathbf{H}_{k}$ is a MIMO channel with independent, uncorrelated and unit variance Gaussian elements.

As the model (4) is calculated from the channel with uncorrelated coefficients, it would be natural to try to formulate the time evolution of spatially correlated channels in a way analog to (3). This is done in [9] for a channel model different from (4). Unfortunately, the parameters of the AR model in [9] are calculated from channel measurements and are not presented in the paper. Therefore, to obtain an AR model for time-varying, spatially correlated MIMO channels, we can start by applying the $\operatorname{vec}(\cdot)$ operator to (4)

$$
\operatorname{vec}\left(\mathbf{H}_{k}^{\mathrm{c}}\right)=\mathbf{h}_{k}^{\mathrm{c}}=\left(\mathbf{R}_{T}^{1 / 2} \otimes \mathbf{R}_{R}^{1 / 2}\right) \operatorname{vec}\left(\mathbf{H}_{k}\right)=\mathbf{G h}_{k},
$$

where $\mathbf{G}=\mathbf{R}_{T}^{1 / 2} \otimes \mathbf{R}_{R}^{1 / 2}, \otimes$ represents the Kronecker product and we use the fact that, for any matrices $\mathbf{D}=\mathbf{A B C}$ of compatible sizes, vec $(\mathbf{D})=\left(\mathbf{C}^{\top} \otimes \mathbf{A}\right) \operatorname{vec}(\mathbf{B})$ [15].

Pre-multiplying (3) by $\mathbf{G}$ results in

$$
\mathbf{G h}_{k}=\beta \mathbf{G h}_{k-1}+\mathbf{G w}_{k} .
$$

Comparing (6) to (5), we can describe the dynamics of spatially correlated MIMO channels as

$$
\mathbf{h}_{k}^{\mathrm{c}}=\beta \mathbf{h}_{k-1}^{\mathrm{c}}+\mathbf{G w}_{k} .
$$


This model is similar to (3), but in (7) the excitation noise is correlated.

\section{Reduced Complexity Channel Estimator}

In order to formulate the problem of channel estimation as one of state estimation, we need two equations named process and measurement equations, respectively [6], [7], [16]. The process equation describes the dynamic behavior of the state variables to be estimated, while the measurement equation presents the relationship between the state variables and the observed system output. As we focus on channel tracking, we can use (7) as the process equation and $\mathbf{h}_{k}$ as the state vector. The system output, in our case, is the channel output $\mathbf{Y}_{k}$ in (1). Thus, the measurement equation can be formed by stacking the columns of $\mathbf{Y}_{k}, \mathbf{H}_{k}$ and $\mathbf{N}_{k}$ in (1), resulting in

$$
\mathbf{y}_{k}=\mathcal{X}_{k} \mathbf{h}_{k}^{\mathrm{c}}+\mathbf{n}_{k},
$$

where $\mathcal{X}_{k}=\mathbf{X}_{k}^{\top} \otimes \mathbf{I}_{N_{R}}$ and $\mathbf{R}_{n}=\sigma_{n}^{2} \mathbf{I}$ is the covariance matrix of the measurement noise $\mathbf{n}_{k}$.

The state-space formulation of the problem of estimating flat, time-varying and spatially correlated MIMO channels is then given by (7) and (8). As both (7) and (8) are linear functions of the state vector $\mathbf{h}_{k}$ and the noises $\mathbf{w}_{k}$ and $\mathbf{n}_{k}$ are independent, white and Gaussian, the Kalman filter provides the optimal recursive estimates, in the MMSE sense, for the channel coefficients [6], [7]. The classical KF for estimating the MIMO channel is given by

$$
\begin{gathered}
\hat{\mathbf{h}}_{k \mid k-1}^{\mathrm{c}}=\beta \hat{\mathbf{h}}_{k-1 \mid k-1}^{\mathrm{c}} \\
\mathbf{P}_{k \mid k-1}=\beta^{2} \mathbf{P}_{k-1 \mid k-1}+\mathbf{G Q G}^{\mathrm{H}} \\
\mathbf{K}_{k}=\mathbf{P}_{k \mid k-1} \mathcal{X}_{k}^{\mathrm{H}}\left(\boldsymbol{\mathcal { X }}_{k} \mathbf{P}_{k \mid k-1} \mathcal{X}_{k}^{\mathrm{H}}+\mathbf{R}_{n}\right)^{-1} \\
\boldsymbol{\alpha}_{k}=\mathbf{y}_{k}-\boldsymbol{\mathcal { X }}_{k} \hat{\mathbf{h}}_{k \mid k-1}^{\mathrm{c}} \\
\hat{\mathbf{h}}_{k \mid k}^{\mathrm{c}}=\hat{\mathbf{h}}_{k \mid k-1}^{\mathrm{c}}+\mathbf{K}_{k} \boldsymbol{\alpha}_{k} \\
\mathbf{P}_{k \mid k}=\left[\mathbf{I}-\mathbf{K}_{k} \mathcal{X}_{k}\right] \mathbf{P}_{k \mid k-1} .
\end{gathered}
$$

In these equations, $\mathbf{P}_{i \mid j}$ is the estimation error covariance matrix at a block $i$ given the signals received from the beginning until block $j$ and $\hat{\mathbf{h}}_{i \mid j}^{\mathrm{c}}$ is the vector with estimated channel coefficients, at a data block $i$, computed from the observation until block $j$.

As mentioned in section II, one of the main properties of any OSTBC is the orthogonality of the codewords. This characteristic is still valid for the transformed codeword $\mathcal{X}_{k}$, as shown in the following lemma:

Lemma 1: The matrix $\boldsymbol{X}_{k}$ satisfies

$$
\mathcal{X}_{k}^{\mathrm{H}} \boldsymbol{\mathcal { X }}_{k}=\left\|\mathbf{x}_{k}\right\|^{2} \mathbf{I}_{N_{R} N_{T}} .
$$

Proof: Given that $\mathbf{X}_{k}$ is an OSTBC and using properties of Kronecker products [15], we can write

$$
\begin{aligned}
\mathcal{X}_{k}^{\mathrm{H}} \boldsymbol{\mathcal { X }}_{k} & =\left(\mathbf{X}_{k}^{\top} \otimes \mathbf{I}_{N_{R}}\right)^{\mathrm{H}}\left(\mathbf{X}_{k}^{\top} \otimes \mathbf{I}_{N_{R}}\right)=\mathbf{X}_{k}^{*} \mathbf{X}_{k}^{\top} \otimes \mathbf{I}_{N_{R}} \\
& =\left(\mathbf{X}_{k} \mathbf{X}_{k}^{\mathrm{H}}\right)^{\top} \otimes \mathbf{I}_{N_{R}}=\left\|\mathbf{x}_{k}\right\|^{2} \mathbf{I}_{N_{T}} \otimes \mathbf{I}_{N_{R}} \\
& =\left\|\mathbf{x}_{k}\right\|^{2} \mathbf{I}_{N_{R} N_{T}},
\end{aligned}
$$

where $(\cdot)^{*}$ is the conjugate of a matrix.

The complexity of (9a)-(9f) can be reduced by exploiting the orthogonality of the transformed OSTBC codewords $\mathcal{X}_{k}$ as well as the model characteristics. As shown in the appendix, the resulting reduced complexity Kalman channel estimator (KCE) for correlated MIMO-OSTBC systems is given by

$$
\begin{aligned}
\mathbf{P}_{k \mid k-1} & =\beta^{2} \mathbf{P}_{k-1 \mid k-1}+\sigma_{w}^{2} \mathbf{R}_{\mathbf{h}} \\
\mathbf{A}_{k} & =\mathbf{P}_{k \mid k-1}\left(\frac{\sigma_{n}^{2}}{\left\|\mathbf{x}_{k}\right\|^{2}} \mathbf{I}_{N_{R} N_{T}}+\mathbf{P}_{k \mid k-1}\right)^{-1} \\
\mathbf{B}_{k} & =\mathbf{I}_{N_{R} N_{T}}-\mathbf{A}_{k} \\
\hat{\mathbf{h}}_{k \mid k}^{\mathrm{c}} & =\beta \mathbf{B}_{k} \hat{\mathbf{h}}_{k-1 \mid k-1}^{\mathrm{c}}+\frac{1}{\left\|\mathbf{x}_{k}\right\|^{2}} \mathbf{A}_{k} \mathcal{X}_{k}^{\mathrm{H}} \mathbf{y}_{k} \\
\mathbf{P}_{k \mid k} & =\mathbf{B}_{k} \mathbf{P}_{k \mid k-1},
\end{aligned}
$$

where $\mathbf{R}_{\mathbf{h}}=\mathrm{E}\left[\mathbf{h}_{k}^{\mathrm{c}} \mathbf{h}_{k}^{\mathrm{c}} \mathrm{H}\right]=\mathbf{G G}^{\mathrm{H}}=\mathbf{R}_{T} \otimes \mathbf{R}_{R}$.

It is worth observing that the channel estimates produced by the proposed Kalman filter (12a)-(12e) correspond to weighted sums of instantaneous ML channel estimates. To see this, first consider the instantaneous ML channel estimates, i.e., the estimates computed by using only the $k^{\text {th }}$ data block, which is given by [12]

$$
\hat{\mathbf{h}}_{k}^{(\mathrm{ML})}=\left(\mathcal{X}_{k}^{\mathrm{H}} \mathcal{X}_{k}\right)^{-1} \mathcal{X}_{k}^{\mathrm{H}} \mathbf{y}_{k} .
$$

For OSTBCs, thanks to lemma 1, (13) reduces to

$$
\hat{\mathbf{h}}_{k}^{(\mathrm{ML})}=\left(\left\|\mathbf{x}_{k}\right\|^{2} \mathbf{I}_{N_{R} N_{T}}\right)^{-1} \mathcal{X}_{k}^{\mathrm{H}} \mathbf{y}_{k}=\frac{1}{\left\|\mathbf{x}_{k}\right\|^{2}} \mathcal{X}_{k}^{\mathrm{H}} \mathbf{y}_{k} .
$$

Thus, using (14) the channel estimate (12d) can be rewritten as

$$
\hat{\mathbf{h}}_{k \mid k}^{\mathrm{c}}=\beta \mathbf{B}_{k} \hat{\mathbf{h}}_{k-1 \mid k-1}^{\mathrm{c}}+\mathbf{A}_{k} \hat{\mathbf{h}}_{k}^{(\mathrm{ML})} .
$$

Consequently, the proposed KF updates the channel estimates through weighted sums of instantaneous ML channel estimates. It is important to note that the weights are time-varying and optimally calculated, in the MMSE sense, for each data block.

The proposed estimator (12a)-(12e) is similar to the one proposed in [11]. However, the state-space model in [11] do not use the transformed codeword $\mathcal{X}_{k}$. Instead, it separates the real and the imaginary parts of all quantities involved in the estimation process. Furthermore, one of the key assumptions to the complexity reduction in [11] is the uncorrelated nature of the channel coefficients. However, for a general spatial correlation matrix $\mathbf{R}_{\mathbf{h}}$, it is not possible to simplify (12a)(12e) even more. For this reason, we take another approach to reduce the complexity of KCE (12a)-(12e), proposing a steady-state Kalman channel estimator in section IV. It will be shown in section IV that the steady-state Kalman channel estimator has a complexity order less than or equal to that of the algorithm in [11] and works also for non-diagonal spatial correlation matrices. 


\section{Steady-State Kalman Channel estimator}

The measurement equation (8) represents a time-varying system, since the matrix $\mathcal{X}_{k}$ changes at each transmitted data block. In the proposed Kalman channel estimator (12a) (12e), however, only (12d) has an explicit dependence on $\boldsymbol{\mathcal { X }}_{k}$. Because of the orthogonality of OSTBC codewords, all other expressions in the proposed recursive estimator depend only on the energy of the uncoded data block, i.e. $\left\|\mathbf{x}_{k}\right\|^{2}$. However, for constant modulus signal constellations such as M-PSK, $\left\|\mathbf{x}_{k}\right\|^{2}$ is a constant. In this case, (12a)-(12c) and (12e) are just functions of the initial estimate of $\mathbf{P}_{k \mid k}$, the normalized Doppler rate, the spatial correlation matrix, a constant equal to the energy of the constellation symbols and the variance of the measurement noise.

These parameters can be estimated ahead of time using, for example, the methods proposed in [17] and in the references therein. Thus, we assume that the parameters in (12a)-(12c) and (12e) are known. Furthermore, we can analyze the statespace model (7) and (8) to check if the matrices $\mathbf{P}_{k \mid k}, \mathbf{A}_{k}$ and $\mathbf{B}_{k}$ converge to steady-state values. If this is the case, and if these values can be found, the time-varying matrices could be replaced by constant matrices, originating a low complexity sub-optimal estimator known as the steady-state Kalman filter. As pointed out in [6], the steady-state filter often performs nearly as well as the optimal time-varying filter.

To determine the steady-state Kalman filter, we begin by substituting (12e) into (12a), which yields

$$
\mathbf{P}_{k \mid k-1}=\beta^{2} \mathbf{B}_{k-1} \mathbf{P}_{k-1 \mid k-2}+\sigma_{w}^{2} \mathbf{R}_{\mathbf{h}} .
$$

Now substitute (12c) into (16) to obtain

$$
\mathbf{P}_{k \mid k-1}=\beta^{2}\left(\mathbf{I}_{N_{R} N_{T}}-\mathbf{A}_{k-1}\right) \mathbf{P}_{k-1 \mid k-2}+\sigma_{w}^{2} \mathbf{R}_{\mathbf{h}} .
$$

Taking into account (12b), we can rewrite (17) as

$$
\begin{aligned}
& \mathbf{P}_{k \mid k-1}=\beta^{2} \mathbf{P}_{k-1 \mid k-2}- \\
& \beta^{2} \mathbf{P}_{k-1 \mid k-2}\left(\frac{\sigma_{n}^{2}}{n_{s}} \mathbf{I}_{N_{R} N_{T}}+\mathbf{P}_{k-1 \mid k-2}\right)^{-1} \mathbf{P}_{k-1 \mid k-2}+\sigma_{w}^{2} \mathbf{R}_{\mathbf{h}},
\end{aligned}
$$

where $n_{s}=\|\mathbf{x}\|^{2}$ corresponds to the energy of each uncoded data block $\mathbf{x}_{k}$, assumed to be a constant.

If $\mathbf{P}_{k \mid k-1}$ converges to a steady-state value, then $\mathbf{P}_{k \mid k-1}=$ $\mathbf{P}_{k-1 \mid k-2}$ for large $k$. Denoting this steady-state value as $\mathbf{P}_{\infty}$, we rewrite (18) as

$$
\mathbf{P}_{\infty}=\beta^{2} \mathbf{P}_{\infty}-\beta^{2} \mathbf{P}_{\infty}\left(\mathbf{P}_{\infty}+\frac{\sigma_{n}^{2}}{n_{s}} \mathbf{I}_{N_{R} N_{T}}\right)^{-1} \mathbf{P}_{\infty}+\sigma_{w}^{2} \mathbf{R}_{\mathbf{h}}
$$

Equation (19) is a discrete algebraic Riccati equation (DARE) [6], [7]. Thus, if $\mathbf{P}_{k \mid k-1}$ converges, we can use $\mathbf{P}_{\infty}$ in (12b) and (12c) to calculate the steady-state values of matrices $\mathbf{A}$ and $\mathbf{B}$, denoted $\mathbf{A}_{\infty}$ and $\mathbf{B}_{\infty}$, respectively. Hence, the proposed steady-state Kalman channel estimator (SS-KCE) is given simply by

$$
\hat{\mathbf{h}}_{k \mid k}^{\mathrm{c}}=\beta \mathbf{B}_{\infty} \hat{\mathbf{h}}_{k-1 \mid k-1}^{\mathrm{c}}+\frac{1}{n_{s}} \mathbf{A}_{\infty} \mathcal{X}_{k}^{\mathrm{H}} \mathbf{y}_{k} .
$$

TABLE I

COMPLEXITY ORDERS OF SOME KALMAN CHANNEL ESTIMATORS

\begin{tabular}{|c||c|}
\hline Algorithm & Complexity per iteration \\
\hline \hline KF & $\mathcal{O}\left(N_{R}^{3} T^{3}\right)$ \\
\hline KCE & $\mathcal{O}\left(N_{R}^{3} N_{T}^{3}\right)$ \\
\hline KF [11] & $\mathcal{O}\left(N_{R}^{2} N_{T} T\right)$ \\
\hline SS-KCE & $\begin{cases}\mathcal{O}\left(N_{R} N_{T} T\right), & N_{R}<T / N_{T} ; \\
\mathcal{O}\left(N_{R}^{2} N_{T}^{2}\right), & N_{R} \geq T / N_{T}\end{cases}$ \\
\hline
\end{tabular}

As in (15), the steady-state KF generates channel estimates by averaging instantaneous ML channel estimates. However, as opposed to (15), the weights in (20) are not time-varying.

The computational complexity per iteration, i.e. for each data block, for the proposed algorithms as well as for the traditional KF (9a)-(9f) and for the KF developed in [11] can be seen in Table I. We notice that, as $T \geq N_{T}$ for OSTBCs, the complexity of the proposed KCE is at most of the same order of the traditional KF. We notice also that the complexity per iteration of the Kalman channel estimator presented in [11] is greater than or equal to the order of the proposed SSKCE. It is important to highlight that the complexity reduction obtained in [11] was only possible because there was no spatial correlation between the channel coefficients. On the other hand, the SS-KCE enjoys an even lower computational complexity for a general spatial correlation matrix.

\section{Simulation Results}

In this section, we present some simulation results to illustrate the performance of the proposed channel estimation algorithms. In all simulations the correlated channels are generated by (5), where the elements of $\mathbf{h}_{k}$ are Rayleigh distributed with time autocorrelation function given by (2). It is worth emphasizing that the proposed estimators approximate the channel dynamics by the first order AR model (7). We assume a periodic transmission of a certain number of spacetime training codewords, after which the channel estimators employ the decisions provided by the ML space-time decoder. Note that these decisions are based on the channel estimates generated by the estimation algorithms in the previous iteration. In all simulations described in the sequel, we insert 25 OSTBC training codewords between every 225 OSTBC data codewords.

Supposing that the spatial correlation coefficient between any two adjacent receive (transmit) antennas is given by $p_{r}$ $\left(p_{t}\right)$, it is possible to express each $(i, j)$ element of the spatial correlation matrices $\mathbf{R}_{R}$ and $\mathbf{R}_{T}$ as $p_{r}^{|i-j|}, i, j=1, \ldots, N_{R}$ and $p_{t}^{|i-j|}, i, j=1, \ldots, N_{T}$, respectively. We assume that the receiver has perfect knowledge of the variances of process and measurement noises, the spatial correlation matrix and the normalized Doppler rate $f_{D} T_{s}$. These parameters can be estimated as in [9], [17]. We also use the MATLAB function dare to numerically solve the Riccati equation (19).

To verify if there is any performance degradation of the SS-KCE (20) compared to the KCE (12a)-(12e), we simulate 


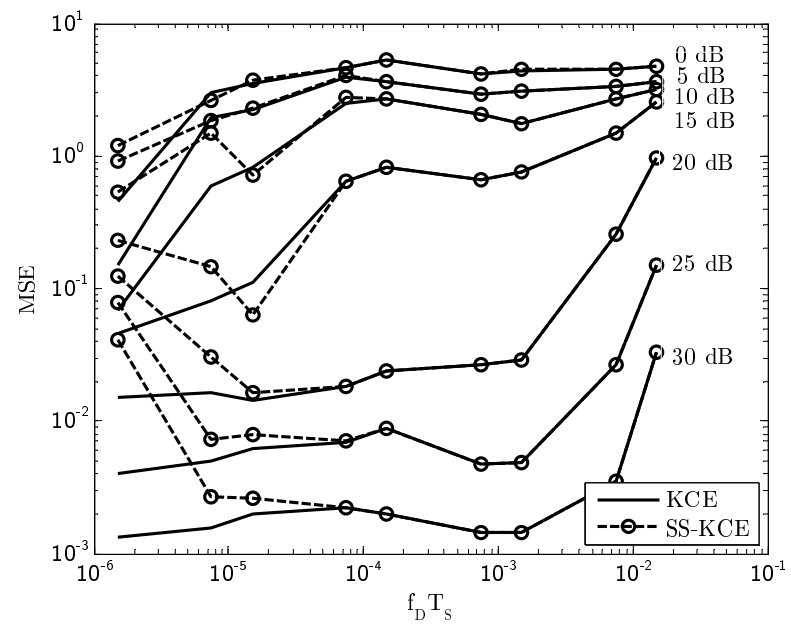

Fig. 1. Estimation mean squared error for KCE and SS-KCE.

the transmission of 8-PSK symbols from $N_{T}=2$ transmit antennas to $N_{R}=2$ receive antennas using the Alamouti space-time block code [1]. We also assume $p_{t}=0.4, p_{r}=0$ and different normalized Doppler rates. Fig. 1 shows the estimation mean squared error (MSE) for KCE and SS-KCE as a function of $f_{D} T_{s}$. We observe that the smaller the value of $f_{D} T_{s}$ (i.e. the smaller the relative velocity between transmitter and receiver), the greater the gap between KCE and SS- KCE. In the limit when $f_{D} T_{s}=0$, the channel is time-invariant, the solution of (19) is null and the SS-KCE does not update the channel estimates. On the other hand, for channels varying at typical rates, both algorithms have equivalent performances. This can be seen in Fig. 2, which presents the symbol error rates at the output of ML space-time decoders fed with channel state information (CSI) provided by $\mathrm{KCE}$ and $\mathrm{SS}-\mathrm{KCE}$, as well as at the output of an ML decoder with perfect channel knowledge. Cleary, SS-KCE has the same performance of the $\mathrm{KCE}$ for the two values of $f_{D} T_{s}$ considered while demanding just a fraction of the complexity.

To illustrate the capacity of the proposed algorithms to track a larger number of channels, we simulate a system sending QPSK symbols from $N_{T}=4$ transmit to $N_{R}=4$ receive antennas. For comparison purposes, we also simulate a channel estimator implemented by the well known RLS adaptive filter [16], with a forgetting factor of 0.98 . This value was determined by trial and error to yield the best performance of the RLS. We employ the $1 / 2$-rate OSTBC of [2] and assume $p_{t}=0.8$ and $p_{r}=0.4$. The MSE for the RLS and the SS-KCE is shown in Fig. 3. We observe that the estimates produced by the RLS algorithm are affected by the rate of channel variation. Moreover, the RLS MSE flattens out for SNR's greater than $10 \mathrm{~dB}$. On the other hand, for this scenario, the proposed SS-KCE has the same performance for both values of $f_{D} T_{s}$ considered and the MSE decreases with the SNR. The similar performances of SS-KCE for $f_{D} T_{s}=0.0015$ and $f_{D} T_{s}=0.0045$ are also reflected in the

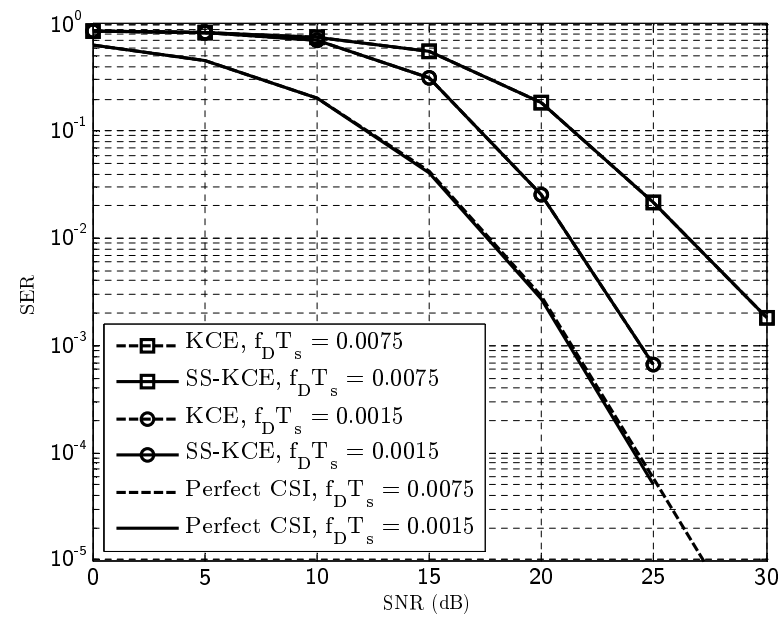

Fig. 2. Symbol error rates of ML decoders fed with channel estimates provided by KCE and SS-KCE. Notice that the curves are not distinguishable.

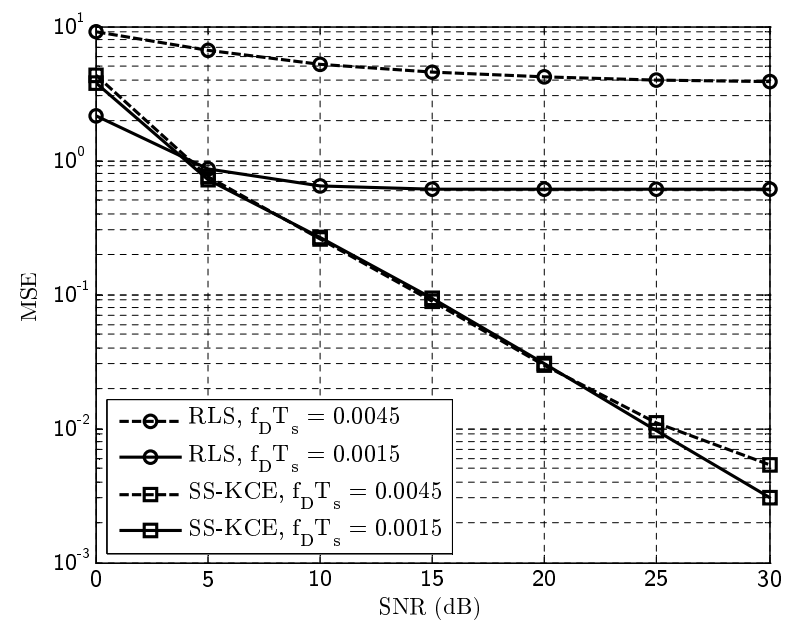

Fig. 3. Estimation mean squared error for different values of $f_{D} T_{s}$.

SER at the output of the ML decoders, as shown in Fig. 4. For an SER of $10^{-3}$, the decoders using the channels estimates provided by the SS-KCE are about $1 \mathrm{~dB}$ from the curves of the ML decoders with perfect CSI. For an SER of $10^{-3}$ and $f_{D} T_{s}=0.0015$ the decoder fed with RLS channels estimates is approximately $4 \mathrm{~dB}$ from the optimal decoder, while for $f_{D} T_{s}=0.0045$ the RLS-based decoder presents an SER no smaller than $10^{-1}$ in the simulated SNR range.

\section{CONCLUSION}

In this paper, we propose channel estimation algorithms intended for systems employing orthogonal space-time block codes. We formulate the problem of channel estimation as one of state estimation and apply the well-known Kalman filter to that state-space model. Then, by using the orthogonality of OSTBCs, we derive a low-complexity optimal Kalman channel estimator. We show that this estimator is a generalization of 


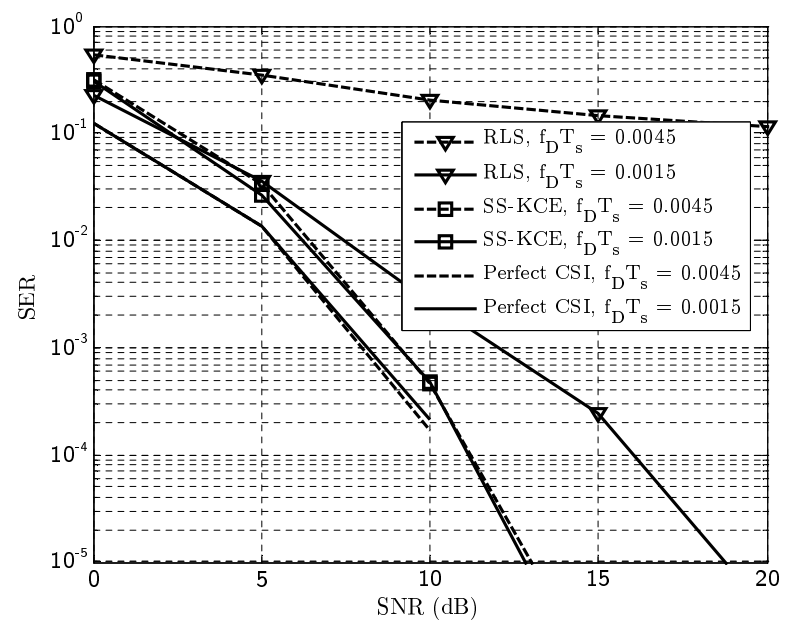

Fig. 4. Symbol error rate for different values of $f_{D} T_{s}$.

previously proposed algorithms and that the channel estimates provided by the KCE in fact correspond to weighted sums of instantaneous ML channel estimates. For constant modulus signal constellations we reduce the receiver complexity even more by proposing a steady-state Kalman filter. This filter also generates channel estimates by averaging instantaneous ML channel estimates.

In terms of computational complexity, the KCE has at most the same complexity order as the traditional Kalman filter. On the other hand, the SS-KCE presents a complexity order smaller than that of others Kalman estimators in the literature. However, the existing methods only work for uncorrelated channels, while the SS-KCE works also for correlated channels. Simulation results indicate that the SS$\mathrm{KCE}$ performs nearly as well as the optimal KCE, while demanding just a fraction of the calculations, and that both algorithms outperform other adaptive filters.

\section{APPENDIX}

In this appendix, we derive the KF in (12a)-(12e). To that end, we begin by substituting $\mathbf{R}_{\mathbf{h}}=\mathbf{G G}^{\mathrm{H}}$ into (9b)

$$
\mathbf{P}_{k \mid k-1}=\beta^{2} \mathbf{P}_{k-1 \mid k-1}+\mathbf{G Q G}^{\mathrm{H}}=\beta^{2} \mathbf{P}_{k-1 \mid k-1}+\sigma_{w}^{2} \mathbf{R}_{\mathbf{h}} \text {. }
$$

Remembering that $\mathbf{R}_{n}=\sigma_{n}^{2} \mathbf{I}_{N_{R} T}$ and using the matrix inversion lemma and (10), it is possible to rewrite (9c) as

$$
\begin{aligned}
& \mathbf{K}_{k}= \\
& \frac{1}{\sigma_{n}^{2}} \mathbf{P}_{k \mid k-1}\left[\mathbf{I}_{N_{R} N_{T}}-\frac{\left\|\mathbf{x}_{k}\right\|^{2}}{\sigma_{n}^{2}}\left(\frac{\left\|\mathbf{x}_{k}\right\|^{2}}{\sigma_{n}^{2}} \mathbf{I}_{N_{R} N_{T}}+\mathbf{P}_{k \mid k-1}^{-1}\right)^{-1}\right] \mathcal{X}_{k}^{\mathrm{H}} .
\end{aligned}
$$

Employing the matrix inversion lemma once more, it is possible to write the inverse matrix of (22) as

$$
\begin{array}{r}
\left(\frac{\left\|\mathbf{x}_{k}\right\|^{2}}{\sigma_{n}^{2}} \mathbf{I}_{N_{R} N_{T}}+\mathbf{I}_{N_{R} N_{T}} \mathbf{P}_{k \mid k-1}^{-1} \mathbf{I}_{N_{R} N_{T}}\right)^{-1}=\frac{\sigma_{n}^{2}}{\left\|\mathbf{x}_{k}\right\|^{2}} \mathbf{I}_{N_{R} N_{T}}- \\
\left(\frac{\sigma_{n}^{2}}{\left\|\mathbf{x}_{k}\right\|^{2}}\right)^{2}\left(\frac{\sigma_{n}^{2}}{\left\|\mathbf{x}_{k}\right\|^{2}} \mathbf{I}_{N_{R} N_{T}}+\mathbf{P}_{k \mid k-1}\right)^{-1}
\end{array}
$$

Substituting now (23) into (22), the Kalman gain adaptation equation is expressed as

$$
\mathbf{K}_{k}=\frac{1}{\left\|\mathbf{x}_{k}\right\|^{2}} \mathbf{A}_{k} \mathcal{X}_{k}^{\mathrm{H}},
$$

where we define

$$
\mathbf{A}_{k}=\mathbf{P}_{k \mid k-1}\left(\frac{\sigma_{n}^{2}}{\left\|\mathbf{x}_{k}\right\|^{2}} \mathbf{I}_{N_{R} N_{T}}+\mathbf{P}_{k \mid k-1}\right)^{-1}
$$

Using lemma 1, (9a), (9d), and (24) into (9e), we have

$$
\hat{\mathbf{h}}_{k \mid k}=\beta \mathbf{B}_{k} \hat{\mathbf{h}}_{k-1 \mid k-1}+\frac{1}{\left\|\mathbf{x}_{k}\right\|^{2}} \mathbf{A}_{k} \mathcal{X}_{k}^{\mathrm{H}} \mathbf{y}_{k},
$$

where $\mathbf{B}_{k}$ is defined as

$$
\mathbf{B}_{k}=\mathbf{I}_{N_{R} N_{T}}-\mathbf{A}_{k} .
$$

Finally, using (24) into (9f) we obtain

$$
\mathbf{P}_{k \mid k}=\left(\mathbf{I}_{N_{R} N_{T}}-\mathbf{A}_{k}\right) \mathbf{P}_{k \mid k-1}=\mathbf{B}_{k} \mathbf{P}_{k \mid k-1} .
$$

Hence, the proposed reduced complexity Kalman channel estimator (KCE) for correlated MIMO-OSTBC systems is given by (21), (25), (26), (27) and (28).

\section{REFERENCES}

[1] S. M. Alamouti, "A Simple Transmit Diversity Technique for Wireless Communications," IEEE J. Sel. Areas Commun., vol. 16, no. 10, pp. 1451-1458, Oct. 1998.

[2] V. Tarokh, H. Jafarkhani, and A. R. Calderbank, "Space-Time Block Codes from Orthogonal Designs," IEEE Trans. Inf. Theory, vol. 45, no. 5, pp. 1456-1467, Jul. 1999.

[3] E. Larsson and P. Stoica, Space-Time Block Coding for Wireless Communications. Cambridge University Press, 2003.

[4] T. M. Duman and A. Ghrayeb, Coding for MIMO Communication Systems. John Wiley and Sons, 2007.

[5] E. Larsson, P. Stoica, and J. Li, "Orthogonal Space-Time Block Codes: Maximum Likelihood Detection for Unknown Channels and Unstructured Interferences," IEEE Trans. Signal Process., vol. 51, no. 2, pp. 362-372, Feb. 2003.

[6] D. Simon, Optimal State Estimation - Kalman, $H_{\infty}$, and Nonlinear Approaches. John Wiley and Sons, 2006.

[7] T. Kailath, A. H. Sayed, and B. Hassibi, Linear Estimation. Prentice Hall, 2000.

[8] R. J. Piechocki, A. R. Nix, J. P. McGeehan, and S. M. D. Armour, "Joint Blind and Semi-Blind Detection and Channel Estimation for SpaceTime Trellis Coded Modulation Over Fast Faded Channels," IEE Proc. Commun., vol. 150, no. 6, pp. 419-426, Dec. 2003.

[9] M. Enescu, T. Roman, and V. Koivunen, "State-Space Approach to Spatially Correlated MIMO OFDM Channel Estimation," Signal Process., vol. 87, no. 9, pp. 2272-2279, Sep. 2007.

[10] Z. Liu, X. Ma, and G. B. Giannakis, "Space-Time Coding and Kalman Filtering for Time-Selective Fading Channels," IEEE Trans. Commun., vol. 50, no. 2, pp. 183-186, Feb. 2002.

[11] B. Balakumar, S. Shahbazpanahi, and T. Kirubarajan, "Joint MIMO Channel Tracking and Symbol Decoding Using Kalman Filtering," IEEE Trans. Signal Process., vol. 55, no. 12, pp. 5873-5879, Dec. 2007.

[12] T. Kaiser, A. Bourdoux, H. Boche, J. R. Fonollosa, J. B. Andersen, and W. Utschick, Eds., Smart Antennas - State of the Art. Hindawi Publishing Corporation, 2005.

[13] M. C. Jeruchim, P. Balaban, and K. S. Shanmugan, Simulation of Communication Systems, 2nd ed. Kluwer Academic, 2000.

[14] F. R. Gantmacher, The Theory of Matrices. AMS Chelsea Publishing, 1959 , vol. 1.

[15] R. A. Horn and C. R. Johnson, Topics in Matrix Analysis. Cambridge University Press, 1991.

[16] S. Haykin, Adaptive Filter Theory, 4th ed. Prentice-Hall, 2002.

[17] A. Jamoos, E. Grivel, W. Bobillet, and R. Guidorzi, "Errors-In-VariablesBased Approach for the Identification of AR Time-Varying Fading Channels," IEEE Signal Process. Lett., vol. 14, no. 11, pp. 793-796, Nov. 2007. 